\title{
Exploring T Cell Reactivity to Gliadin in Young Children with Newly Diagnosed Celiac Disease
}

\author{
Edwin Liu, ${ }^{1}$ Kristen McDaniel, ${ }^{1}$ Stephanie Case, ${ }^{1}$ Liping Yu, ${ }^{1}$ Bernd Gerhartz, ${ }^{2}$ \\ Nils Ostermann, ${ }^{2}$ Gabriela Fankhauser, ${ }^{2}$ Valerie Hungerford, ${ }^{2}$ Chao Zou, ${ }^{2}$ \\ Marcel Luyten, ${ }^{2}$ Katherine J. Seidl, ${ }^{2}$ and Aaron W. Michels ${ }^{1}$ \\ ${ }^{1}$ Barbara Davis Center for Childhood Diabetes, School of Medicine, University of Colorado Denver, \\ Mail Stop A140, 1775 Aurora Court, Aurora, CO 80045, USA \\ ${ }^{2}$ Novartis Institutes for Biomedical Research, 4002 Basel, Switzerland \\ Correspondence should be addressed to Aaron W. Michels; aaron.michels@ucdenver.edu
}

Received 18 November 2013; Revised 15 January 2014; Accepted 16 January 2014; Published 3 March 2014

Academic Editor: Raffaella Nenna

Copyright (C) 2014 Edwin Liu et al. This is an open access article distributed under the Creative Commons Attribution License, which permits unrestricted use, distribution, and reproduction in any medium, provided the original work is properly cited.

\begin{abstract}
Class II major histocompatibility molecules confer disease risk in Celiac disease (CD) by presenting gliadin peptides to CD4 T cells in the small intestine. Deamidation of gliadin peptides by tissue transglutaminase creates immunogenic peptides presented by HLA-DQ2 and DQ8 molecules to activate proinflammatory CD4 T cells. Detecting gliadin specific T cell responses from the peripheral blood has been challenging due to low circulating frequencies and heterogeneity in response to gliadin epitopes. We investigated the peripheral $\mathrm{T}$ cell responses to alpha and gamma gliadin epitopes in young children with newly diagnosed and untreated CD. Using peptide/MHC recombinant protein constructs, we are able to robustly stimulate CD4 T cell clones previously derived from intestinal biopsies of CD patients. These recombinant proteins and a panel of $\alpha$ - and $\gamma$-gliadin peptides were used to assess $\mathrm{T}$ cell responses from the peripheral blood. Proliferation assays using peripheral blood mononuclear cells revealed more CD4 T cell responses to $\alpha$-gliadin than $\gamma$-gliadin peptides with a single deamidated $\alpha$-gliadin peptide able to identify $60 \%$ of CD children. We conclude that it is possible to detect $\mathrm{T}$ cell responses without a gluten challenge or in vitro stimulus other than antigen, when measuring proliferative responses.
\end{abstract}

\section{Introduction}

Celiac disease $(\mathrm{CD})$ is a T cell mediated enteropathy triggered by the ingestion of dietary gluten resulting in villous atrophy and crypt hyperplasia in the small intestine [1]. Specific human leukocyte antigen (HLA) genes are involved in the disease process with restriction primarily limited to HLA-DQ2 (DQA $* 05: 01, \mathrm{DQB} * 02: 01$ and $\mathrm{DQA} * 02: 01$, $\mathrm{DQB} * 02: 02)$ and $\mathrm{DQ} 8(\mathrm{DQA} * 03: 01, \mathrm{DQB} * 3: 02)$ [2]. HLA genes encode class II major histocompatibility molecules (MHC), which present antigens to CD4 T cells. There has been remarkable progress in the understanding of the pathogenesis and epitopes involved in the disease process [3-6]. Gliadin, one of the two principle protein components of gluten, contains a number of well-studied $\mathrm{T}$ cell epitopes. Deamidation of gliadin peptides by tissue transglutaminase
(TTG) type 2 converts glutamine into glutamic acid, resulting in immunogenic T cell epitopes [7].

Despite the current understanding of T cell-peptideMHC interaction, simple and reliable T cell assays from the peripheral blood to monitor $\mathrm{CD}$ activity have been difficult to develop. Peripheral blood biomarkers for CD are hindered by variables such as the type of assay, use of the proper antigen, low circulating frequencies of $\mathrm{T}$ cells, and the timing of gluten exposure when the assay is performed. For example, it has been reported that, to obtain sufficient $\mathrm{T}$ cells for study without in vitro expansion, CD blood donors on a gluten-free diet need to undergo short-term gluten challenge, to detect IFN- $\gamma$ T cell responses by enzyme linked immunospot (ELISPOT) assays [8, 9]. However, both glutenfree and gluten-exposed CD patients can have measurable $\mathrm{T}$ cell proliferative responses with response rates more 
frequent in gluten-exposed patients [10]. Comprehensive epitope mapping studies have identified four immunodominant DQ2 epitopes in treated CD adults (gluten-free diet) followed by a gluten challenge [11]. Such an unbiased epitope mapping project has not been undertaken for children with $\mathrm{CD}$, even though there are several well-studied epitopes in the literature.

In this study, we investigated the peripheral $\mathrm{T}$ cell responses to alpha and gamma gliadin epitopes in young children with newly diagnosed and untreated CD. The selected epitopes are known to stimulate $\mathrm{T}$ cell clones derived from adult CD patient intestinal biopsies [12, 13]. Recombinant DQ2 and DQ8 proteins with $\alpha$-gliadin epitopes were created to test $\mathrm{T}$ cell clone stimulation without the use of antigen presenting cells, as part of an effort to develop a $\mathrm{T}$ cell stimulation assay feasible for large scale, consistent, and rapid assessment of $\mathrm{CD}$ activity. We explored the utility of peptide/MHC complexes first in stimulating $\mathrm{T}$ cell clones and then peripheral blood mononuclear cells (PBMCs) of newly diagnosed $\mathrm{CD}$ children prior to treatment with a gluten-free diet to produce inflammatory cytokines. Subsequently, proliferation assays utilizing a panel of previously described DQ2 and DQ8 peptides on the same CD children provide insight into the possible degenerate nature of $\alpha$-gliadin peptide binding motifs for HLA-DQ2.

\section{Methods}

2.1. Subjects and Samples. Subjects were recruited from the Children's Hospital Colorado, and written informed consent was obtained after the nature and possible consequences of the study were explained to individuals. The clinical investigation in this study was conducted in accordance with the Declaration of Helsinki principles, and study approval was provided by the Colorado Multiple Institutional Review Board. Peripheral blood was obtained for T cell assays, TTG antibodies, and HLA genotyping. TTG antibodies were measured from the serum by radioimmunoassay as previously described [14]. HLA-DRB, DQA, and DQB genotyping were performed using linear arrays of immobilized sequencespecific oligonucleotides similar to previously described methodology [15].

\subsection{Expression and Purification of Recombinant Protein. The} extracellular domains of the HLA-DQ8 $\alpha$ chain (residue 24-204) and $\beta$ chain (residue 33-221) were coexpressed in S2 drosophila cells (ExpreS2ion Biotechnologies, Denmark). Double deamidated $\alpha$-gliadin (QQYPSGEGSFQPSQENPQ) was covalently attached to the N-terminus of $\beta$ chain with a Factor $X$ cleavage site (GGGGSIEGRGSGGGS) between the peptide and the $\beta$ chain. To stabilize the heterodimer, Fos and Jun leucine zippers were attached to the C-terminus of $\alpha$ chain and $\beta$ chain via a thrombin cleavage sequence (SSADLVPRGS). Deamidated $\alpha$-gliadin/DQ8 was extracted from the medium using anti-FLAG M2 (Sigma Aldrich) affinity chromatography. The recombinant protein was purified by Superdex 200 column in buffer containing 10 mMTris, $\mathrm{pH}$ 8.0 , and $150 \mathrm{mM} \mathrm{NaCl}$. For the $\alpha$-I-gliadin/DQ2 construct, the extracellular domains of HLA-DQ2 $\alpha$ chain (residue 24206 ) and $\beta$ chain (residue 33-221) were used, expressed in S2 drosophila cells, and purified in a similar manner to the DQ8 recombinant protein construct. The amino acid sequence of $\alpha$-I gliadin, QLQPFPQPELPY, was covalently attached to the $\mathrm{N}$-terminus of the $\beta$ chain via TEV cleavage site (GGGGENLYFQGGSGGGS). To stabilize the heterodimer, Fos and Jun leucine zippers were attached via PreScission cleavage site (SSADLEVLFQGP) to the C-termini of $\alpha$ chain and $\beta$ chain, respectively. The final proteins were confirmed by LC-MS. Diagrams of the two recombinant protein constructs are depicted in Figure 1(a).

2.3. Generation of T Cell Receptor Hybridomas. T cell receptor (TCR) hybridomas, containing the TCR from a DQ2 and DQ8 restricted T cell clone responding to $\alpha$-gliadin peptides, were created as previously described [16]. The sequences for the $\alpha \beta$ TCR clones in Figure 2 were a kind gift from Ludvig Sollid. The sequences for the $\alpha \beta$ TCR clones in the supplemental Figures (see supplementary material available online at http://dx.doi.org/10.1155/2014/927190) were derived from published information on gene usage [13]. Briefly, a single TCR sequence, $\alpha$ and $\beta$ chains linked by the PTV1.2A sequence, was cloned into MSCV-based retroviral vectors carrying green fluorescent protein (GFP) (pMIGII) [17], followed by production of replication-incompetent retroviruses encoding TCR sequences. The $5 \mathrm{KC}$ hybridoma line lacking TCR $\alpha$ and $\beta$ chains was used to reconstitute TCRs [18]. The transduced 5KC hybridomas were sorted by GFP expression and TCR expression was confirmed by staining with anti-mouse TCR $\beta$ antibody (clone H57-597, BD Biosciences). Alternatively, the expression of plasmids in the pMSCVpuro retroviral vector (Clontech) was transfected to AmphoPack-293 or GP2-293 (VSV-G envelope) packaging cells (Clontech) to produce retrovirus and mouse $5 \mathrm{KC}$ cells were spin-infected with retroviral supernatants and cultured with puromycin. TCR expression was confirmed in isolated single clones by staining with anti-mouse CD3 (clone 1452C11; BD Biosciences) or mouse TCR $\beta$ antibody (clone H57597; BD Biosciences).

2.4. Cytokine Stimulation Assays. Peripheral blood mononuclear cells (PBMCs) were isolated from whole blood using Ficoll-Paque and resuspended at a density of $10^{6} / \mathrm{mL}$ in IMDM-C media (IMDM supplemented with 5\% heat inactivated human $\mathrm{AB}$ serum, $100 \mu \mathrm{g} / \mathrm{mL}$ Pen-Strep, $100 \mu \mathrm{M}$ MEM NEAA, and $50 \mu \mathrm{M} 2$-mercaptoethanol). $2 \times 10^{5}$ PBMCs or T cell hybridomas in $200 \mu \mathrm{L}$ of media were added to a 96well tissue culture plate coated with $1 \mu \mathrm{g} /$ well of recombinant protein and cultured at $37^{\circ} \mathrm{C}$ in $5 \% \mathrm{CO}_{2}$ overnight. Secreted cytokine was measured in the supernatant by electrochemiluminescence assay (Meso Scale Discovery) for human IFN$\gamma$, IL-2, and by ELISA for IL-17 (R\&D Systems). PBMCs in culture without protein (background) were a negative control, while anti-CD3 stimulation (OKT3, eBioscience) was a positive control. DQ antibody SPV-L3 (Abcam, Cambridge, UK), 1a3 (Leinco, St. Louis, MO, USA), or HB-144 (ATCC, Manassas, VA, USA) was added at either $10 \mu \mathrm{g} / \mathrm{mL}$ or at 


\begin{tabular}{|c|c|c|c|c|c|c|}
\hline & & l peptide & DQ alpha chain & Thrombin & Basic leu zipper & FLAG \\
\hline Signal peptide & Peptide & Factor $X$ & DQ beta chain & Thrombin & Acidic leu zipper & His \\
\hline
\end{tabular}

(a)

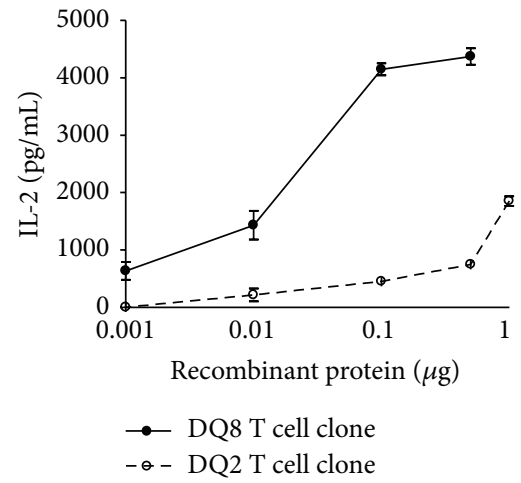

(b)

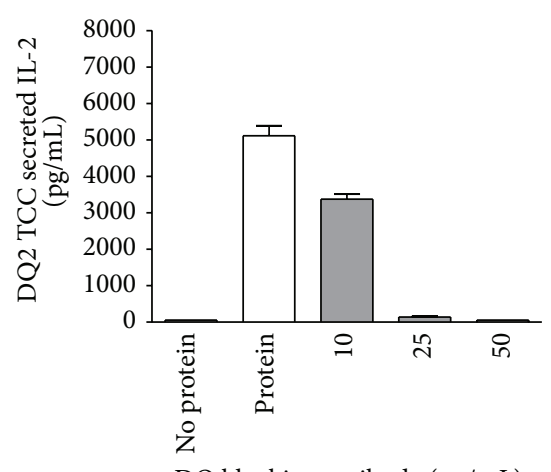

(c)

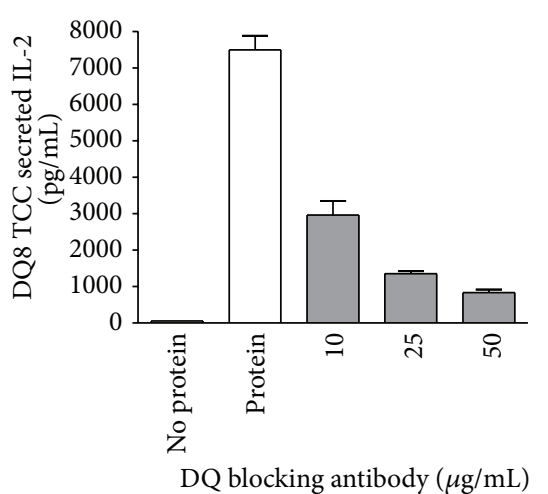

(d)

FIGURE 1: Recombinant DQ8 and DQ2 proteins with gliadin epitopes stimulate T cell clones. (a) Diagrams of the constructs used to produce recombinant protein for deamidated $\alpha$-gliadin p1E, p9E/DQ8, and $\alpha$-I gliadin/DQ2. The amino acid sequence of the $\alpha$-gliadin peptide in DQ8 is QQYPSGEGSFQPSQENPQ and the $\alpha$-I gliadin peptide (QLQPFPQPELPY) with DQ2. Thrombin, TEV, and PreScission are protease cleavage sites incorporated into the protein constructs. (b) T cell clones restricted to either DQ8 or DQ2 produce IL-2 in response to the deamidated $\alpha$-gliadin/DQ8 or $\alpha$-I gliadin/DQ2 recombinant protein, respectively. (c) The DQ2 and (d) DQ8 T cell responses can be blocked in a dose dependent manner with a monoclonal DQ antibody.

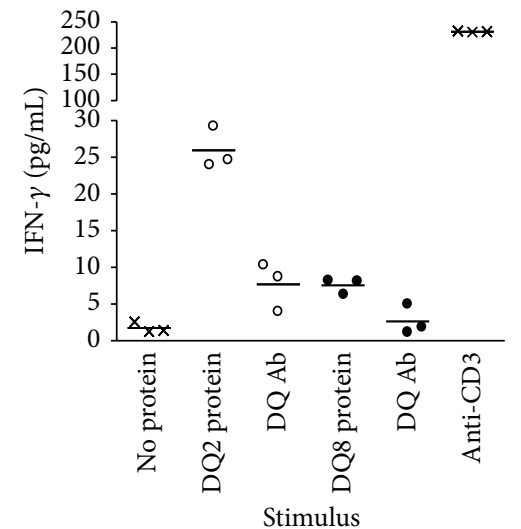

- DQ2 T cell response

- DQ8 T cell response

(a)

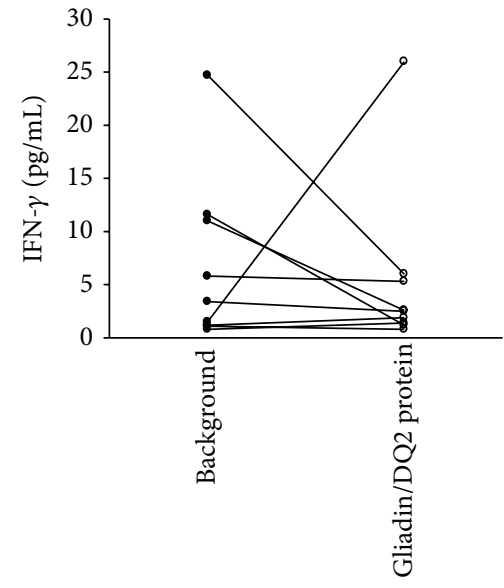

(b)

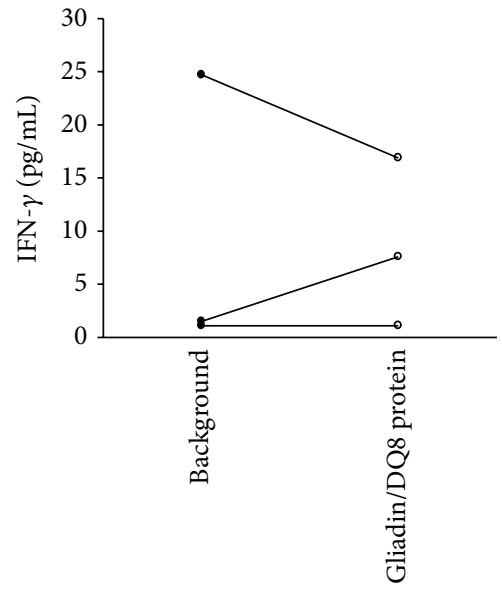

(c)

FIGURE 2: Recombinant peptide/MHC protein stimulates IFN- $\gamma$ production from bulk unfractionated PBMCs. (a) Stimulation of PBMCs from a single subject in triplicate having both HLA-DQ8 and DQ2 alleles showing response to the recombinant proteins greater than background. The IFN- $\gamma$ response is DQ restricted as it can be blocked with a monoclonal DQ antibody. An anti-CD3 monoclonal antibody is used to stimulate T cells as a positive control. The $\alpha$-gliadin p1E, p9E peptide (QQYPSGEGSFQPSQENPQ) is present in the DQ8 recombinant protein, while $\alpha$-I gliadin (QLQPFPQPELPY) is present in the DQ2 protein. (b) Summative stimulation data from nine subjects all with HLADQ2 (DQA *05:01, DQB*02:01). (c) Data from three subjects having the HLA-DQ8 (DQA*03:01, DQB*03:02) allele.

varying concentrations for blocking experiments. All study subjects had cytokine stimulation assays performed.

\subsection{CFSE Proliferation Assay. Isolated and unfractionated} PBMCs were suspended at a density of $10^{6} / \mathrm{mL}$ in CFSE labeling buffer (1\% BSA in PBS). Cells were labeled with $1 \mu \mathrm{M}$ CFSE (eBioscience) for 10 minutes at $37^{\circ} \mathrm{C}$. Labeling was quenched by adding chilled IMDM-C media at 5 times the volume at $0^{\circ} \mathrm{C}$; cells were then incubated on ice for 5 minutes. Labeled cells were washed in PBS with $1 \%$ human AB serum, resuspended in media, and plated into a 24 -well tissue culture plate at $10^{6}$ cells/well in $1 \mathrm{~mL}$ of media. Peptides (Genemed Synthesis Inc.) were HPLC purified (>95\%), dissolved in PBS at a neutral $\mathrm{pH}$, and used at a concentration of $10 \mu \mathrm{M}$. Pentacel vaccine (Sanofi Pasteur) was added at $2 \mu \mathrm{L}$ per well as a positive control. After seven days of incubation 
TABLE 1: Clinical characteristics, TTG antibody levels, histology, and HLA genotype of study participants.

\begin{tabular}{|c|c|c|c|c|c|c|c|c|c|c|}
\hline \multirow{2}{*}{ Case } & \multirow{2}{*}{ Age (yrs) } & \multirow{2}{*}{ Sex } & \multirow{2}{*}{ TTG Ab level* } & \multirow{2}{*}{ Histology marsh score } & \multicolumn{6}{|c|}{ HLA DQ and DR alleles } \\
\hline & & & & & DRB1 & DQA1 & DQB1 & DRB2 & DQA2 & DQB2 \\
\hline$\overline{1}$ & 4 & $\mathrm{~F}$ & 0.145 & 2 & 0404 & 0301 & 0302 & 0301 & 0501 & 0201 \\
\hline 2 & 5 & $\mathrm{~F}$ & 0.130 & 0 & 0403 & 0301 & 0302 & 0301 & 0501 & 0201 \\
\hline 3 & 5 & $\mathrm{M}$ & 0.064 & 1 & 0301 & 0501 & 0201 & 1602 & 0501 & 0301 \\
\hline 4 & 5 & M & 0.178 & $3 b$ & 0301 & 0501 & 0201 & 0301 & 0501 & 0201 \\
\hline 5 & 4 & $\mathrm{~F}$ & 1.127 & $3 c$ & 0301 & 0501 & 0201 & 0801 & 0401 & 0402 \\
\hline 6 & 7 & $\mathrm{~F}$ & 0.877 & No biopsy & 0701 & 0201 & 0202 & 1104 & 0501 & 0301 \\
\hline 7 & 7 & $\mathrm{~F}$ & 0.608 & $3 c$ & 0301 & 0501 & 0201 & 1301 & 0103 & 0603 \\
\hline 8 & 7 & $\mathrm{M}$ & 0.755 & $3 b$ & 0301 & 0501 & 0201 & 0701 & 0201 & 0202 \\
\hline 9 & 7 & $\mathrm{~F}$ & 0.461 & $3 b$ & 0301 & 0501 & 0201 & 1501 & 0102 & 0602 \\
\hline 10 & 12 & $\mathrm{~F}$ & 0.624 & $3 b$ & 0701 & 0201 & 0202 & 1101 & 0501 & 0301 \\
\hline 11 & 13 & M & 0.511 & $3 b$ & 0403 & 0301 & 0302 & 0701 & 0201 & 0202 \\
\hline 12 & 9 & $\mathrm{~F}$ & 0.169 & $3 b$ & 0301 & 0501 & 0201 & 0301 & 0501 & 0201 \\
\hline
\end{tabular}

${ }^{*} \mathrm{TTG} \mathrm{Ab} \geq 0.05$ is elevated.

at $37^{\circ} \mathrm{C}$ in $5 \% \mathrm{CO}_{2}$, nonadherent cells were harvested and stained for FACS analysis using antibodies to CD4 (RPA-T4, BD Bioscience) and CD8 (RPA-T8, BD Bioscience). FACS analysis was done using a Becton-Dickenson FACS Caliber. Ten of the 12 study participants gave adequate numbers of PBMCs to perform CFSE proliferation assays.

2.6. Statistical Analysis. The percentage of $\mathrm{CD}^{+} \mathrm{CFSE}^{\text {lo }}$ after proliferation to a given stimulus was compared with a paired Student's $t$-test as conditions were matched in the same subject. Response rates between $\alpha$-gliadin and $\gamma$-gliadin peptides were compared with a two-sided Fisher's exact text. For all statistical tests, a two-tailed $P$ value of $<0.05$ is considered significant. Analyses were performed using GraphPad Prism 4.0 software (La Jolla, CA).

\section{Results}

3.1. Subjects. Subjects with new-onset Celiac disease $(n=12)$ were recruited from the Children's Hospital Colorado Celiac Disease Center clinics. The study protocol was approved by the Institutional Review Board and written informed consent was obtained from all study participants. The Celiac subjects were young children and adolescents, with a mean age of 7.1 years, known to be TTG antibody positive and not on a gluten-free diet prior to having a small intestine biopsy. At the visit for intestinal biopsy, 11/12 (92\%) patients had a biopsy and blood was collected for TTG antibody levels, HLA genotyping, and immune assays. Demographic and clinical characteristics are presented in Table 1. All of the subjects had positive TTG antibody levels and the majority $8 / 12(75 \%)$ had stage 3 Marsh scores on histologic examination of a small intestine biopsy. HLA typing revealed that 9/12 (75\%) subjects had the high risk HLA-DQ2 (DQA1 $* 05: 01, \mathrm{DQB1} * 02: 01)$ allele and 3/12 (25\%) had HLADQ8 (DQA1*03:01, DQB1*03:02) in addition to DQ2.

3.2. T Cell Hybridomas Respond Robustly to Recombinant Peptide/MHC Protein. We produced recombinant peptide/MHC protein to known $\alpha$-gliadin epitopes presented by HLA-DQ2 or DQ8 (Figure 1(a)). The two recombinant proteins, $\alpha$-I
gliadin/DQ2 and deamidated $\alpha$-gliadin p1E, p9E/DQ8, are bioactive and able to robustly stimulate $\mathrm{T}$ cell hybridomas created from CD4 T cells cloned from small intestine lesions of adult CD subjects $[12,13]$. The responses of these $\mathrm{T}$ cell hybridomas, measured by secreted IL-2, are dose dependent and required minimal amounts of protein (less than $1 \mu \mathrm{g} /$ well) for stimulation (Figure 1(b), supplemental Figure 1(a)). The T cell responses are restricted to DQ2 or DQ8, depending on the class II molecule of the recombinant protein, as a DQ monoclonal antibody added in culture was able to abrogate IL-2 secretion (Figures 1(c) and 1(d) and supplemental Figures $1(\mathrm{~b})$ and $1(\mathrm{c}))$.

3.3. Detection of IFN- $\gamma$ Responses to Recombinant Peptide/MHC Proteins. Having recombinant $\alpha$-gliadin/DQ proteins able to robustly stimulate $\mathrm{T}$ cell hybridomas, we evaluated the ability of the recombinant proteins to stimulate $\mathrm{T}$ cell responses from the peripheral blood of newly diagnosed CD subjects. We measured secreted cytokine responses (IFN- $\gamma$, IL-2, and IL-17) after culturing bulk unfractionated PBMCs in the presence of the $\alpha$-I gliadin/DQ2 or $\alpha$-gliadin p1E, p9E/DQ8 recombinant protein. Figure 2(a) depicts IFN $-\gamma$ responses to an individual having both the DQ2 (DQA1 $*$ 05:01, DQB1*02:01) and DQ8 (DQA1 $* 03: 01$, $\mathrm{DQB1} * 03: 02)$ alleles, which are identical to that of the recombinant proteins. There are responses to the protein greater than that of background alone and the responses are blocked by a DQ monoclonal antibody, suggesting that the measured responses are due to reactivity to the $\alpha$-gliadin/DQ proteins. The cumulative data from subjects having corresponding HLA alleles to that of the recombinant protein, however, failed to identify $\mathrm{T}$ cell reactivity above background responses (Figures 2(b) and 2(c)). The measured IL-2 and IL17 responses were not greater than background in any of the subjects (data not shown).

3.4. Proliferation of CD4 T Cells to the $\alpha$ - and $\gamma$-Gliadin Peptides. We next examined $\mathrm{T}$ cell responses to eleven known $\alpha$ - and $\gamma$-gliadin epitopes $[12,19,20]$, in contrast to recombinant peptide/MHC protein, previously identified from CD patients. CD4 $\mathrm{T}$ cell proliferation was assessed from the 

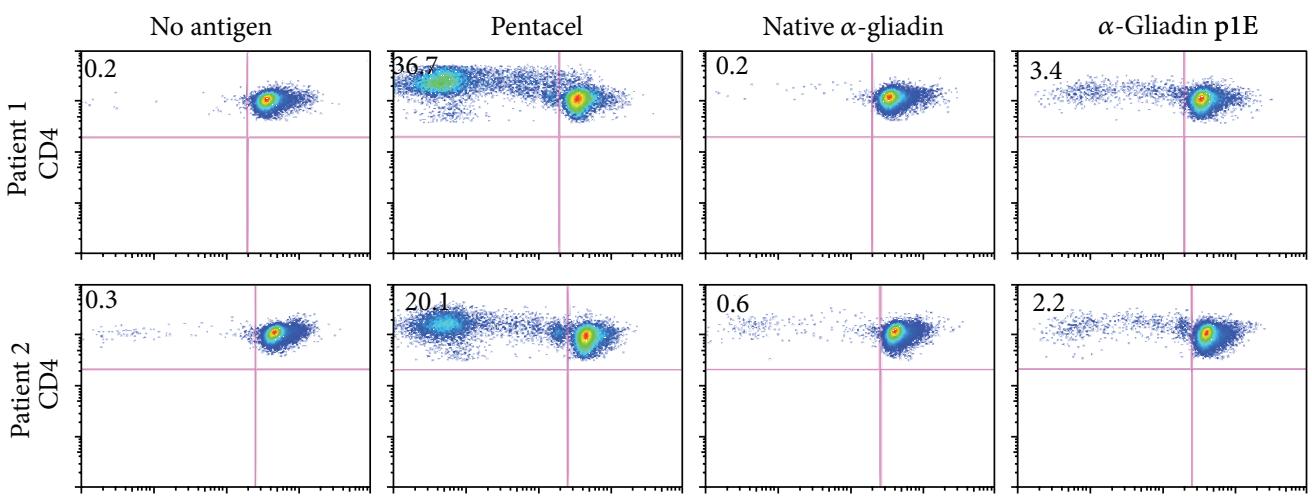

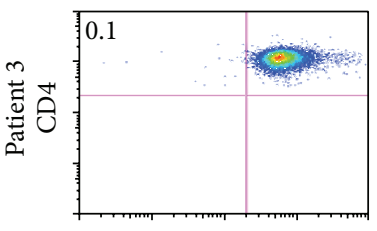

CFSE

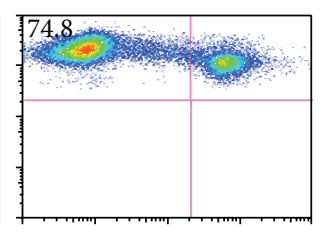

CFSE

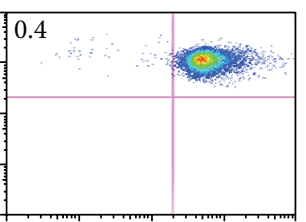

CFSE

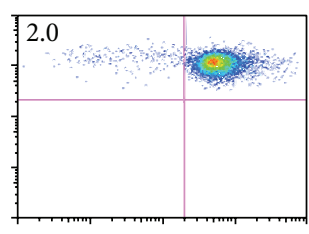

CFSE

(a)

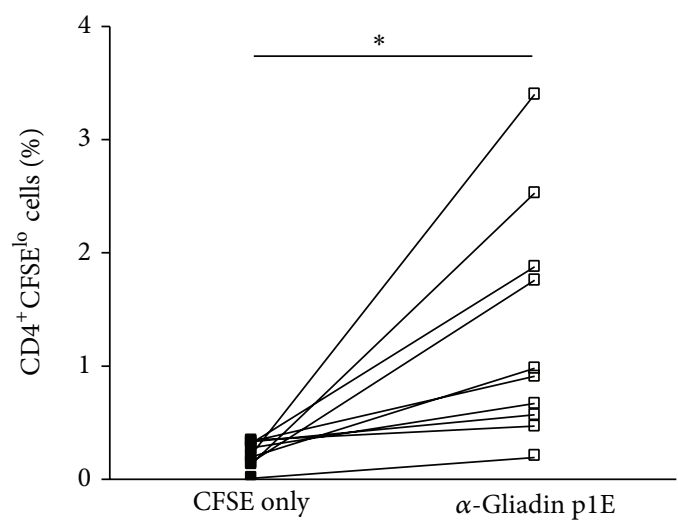

(b)

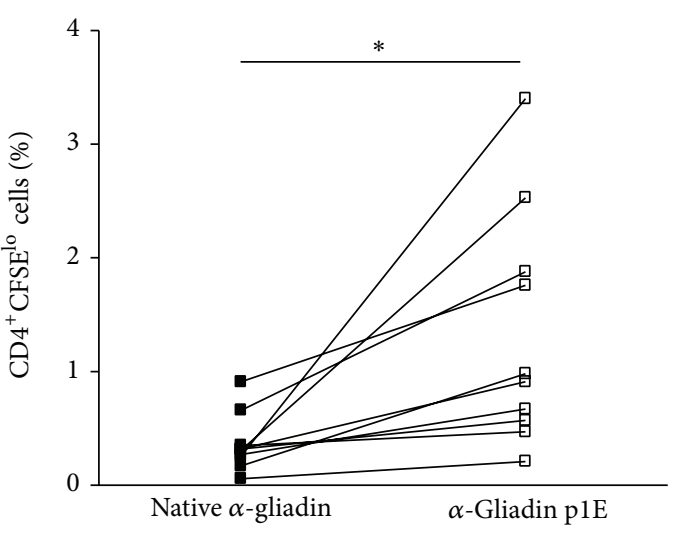

(c)

FIGURE 3: Proliferation of unfractionated PBMCs with $\alpha$-gliadin peptides. (a) Representative data from three newly diagnosed Celiac subjects with 7-day CFSE proliferation assays. CD4 T cells proliferate in response to the $\alpha$-gliadin plE deamidated peptide without the in vitro addition of cytokines. (b) Summary data of proliferative responses comparing CFSE only (no antigen background) to the $\alpha$-gliadin plE peptide. (c) Proliferation of native $\alpha$-gliadin to the $\alpha$-gliadin plE deamidated peptide. ${ }^{*} P<0.01$ using a paired $t$-test. Pentacel (positive control) is a childhood vaccine containing immunogens directed against diphtheria, tetanus, pertussis, poliomyelitis, and Haemophilus influenzae type b.

peripheral blood of newly diagnosed CD subjects. Figure 3 shows the proliferation results after bulk unfractionated PBMCs were labeled with CFSE and cultured for 7 days in the presence of a single gliadin peptide without the addition of any in vitro stimulus, that is, no IL-2, anti-CD3, or anti-CD28. Of the individuals having CFSE proliferation assays performed, there were robust responses to the $\alpha$-gliadin $228-240$ peptide (SGQGSFQPSQQNP), especially with a deamidated glutamate residue at the pocket 1 position. In all of the subjects, the single deamidated peptide (SGEGSFQPSQQNP) resulted in significantly more proliferation as measured by $\mathrm{CD} 4^{+} \mathrm{CFSE}^{\mathrm{lo}}$ cells compared to background (no antigen in culture) and the native $\alpha$-gliadin peptide (Figures $3(\mathrm{~b})$ and $3(c))$. Interestingly, none of the subject's PBMC proliferated in response to the double deamidated $\alpha$-gliadin peptide $(\alpha$-gliadin p1E, p9E) and few responded (3/10) to the $\alpha$-Igliadin peptide, which are the peptides in the DQ8 and DQ2 recombinant protein, respectively (Table 2 ). Evaluating proliferative responses with a stimulation index $\left(\mathrm{CD} 4^{+} \mathrm{CFSE}^{\text {lo }}\right.$ condition $/ \mathrm{CD} 4^{+} \mathrm{CFSE}^{\text {lo }}$ background) greater than 3 revealed $12 / 60(20 \%)$ responses to $\alpha$-gliadin peptides compared to $3 / 50$ (6\%) of $\gamma$-gliadin peptides $(P=0.049)$. Overall, there were more proliferative responses to $\alpha$-gliadin peptides compared to $\gamma$-gliadin in young children with newly diagnosed CD (Figure 4). In those children responding to two or more peptides, all three were HLA-DQ2/2 homozygotes (Table 3). Interestingly, the HLA-DQ2/2 children responded to epitopes which have been previously reported in the literature to be 
TABLE 2: Proliferative responses to $\alpha$ - and $\gamma$-gliadin epitopes.

\begin{tabular}{lcc}
\hline Epitope & Amino acid sequence & Proliferation response** $^{* *}$ \\
\hline Native $\alpha$-gliadin & SGQGSFQPSQQNP & $1 / 10(10 \%)$ \\
$\alpha$-Gliadin plE & SGEGSFQPSQQNP & $6 / 10(60 \%)$ \\
$\alpha$-Gliadin p9E & SGQGSFQPSQENP & $2 / 10(20 \%)$ \\
$\alpha$-Gliadin p1E, p9E & SGEGSFQPSQENP & $0 / 10(0 \%)$ \\
$\alpha$-I-Gliadin & QLQPFPQPELPY & $3 / 10(30 \%)$ \\
$\alpha$-II-Gliadin & PQPELPYPQPQL & $0 / 10(0 \%)$ \\
Native $\gamma$-gliadin & FPQQPQQPYPQQPQQ & $0 / 10(0 \%)$ \\
$\gamma$-Gliadin p1E & FPEQPQQPYPQQPQQ & $1 / 10(10 \%)$ \\
$\gamma$-Gliadin p9E & FPQQPQQPYPEQPQQ & $0 / 10(0 \%)$ \\
$\gamma$-Gliadin p1E, p9E & FPEQPQQPYPEQPQQ & $2 / 10(20 \%)$ \\
$\gamma$-I-Gliadin & PEQPQQSFPEQERP & $0 / 10(0 \%)$ \\
\hline
\end{tabular}

${ }^{*}$ Glutamic acid (E) residues in bold are formed by tissue transglutaminase mediated deamidation. Underlined residues form the MHC class II peptide binding register.

${ }_{* * *}^{*}$ A stimulation index $\geq 3$ is considered a response.

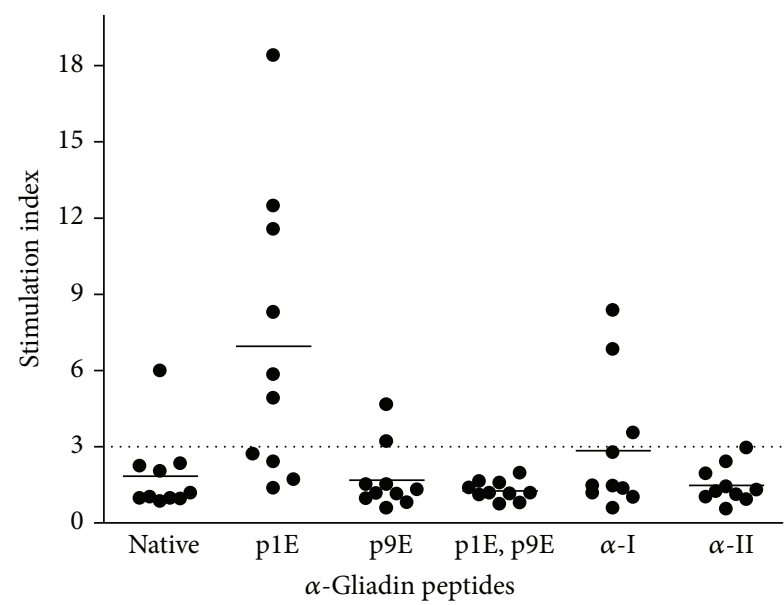

(a)

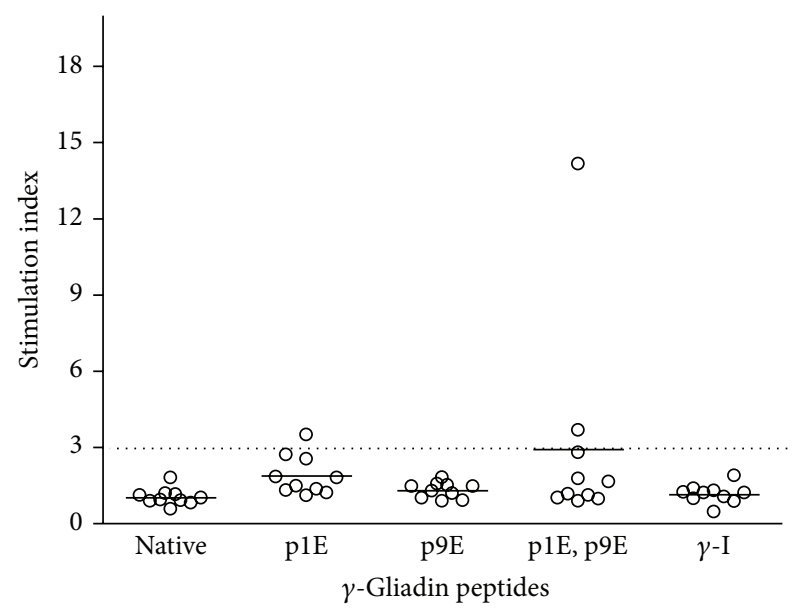

(b)

FIGURE 4: Proliferation of PBMCs from newly diagnosed Celiac patients to $\alpha$ - and $\gamma$-gliadin peptides.(a) Proliferative responses to $\alpha$-gliadin and (b) $\gamma$-gliadin epitopes. Celiac subjects proliferate more in response to $\alpha$-gliadin peptides compared to $\gamma$-gliadin, especially the peptide deamidated at pocket 1 in which 6/10 subjects responded. Overall, there are 12/60 responses to $\alpha$-gliadin peptides compared to $3 / 50$ for $\gamma$ gliadin $\left(P=0.049\right.$ with a Fisher's exact test). Dotted line is at a stimulation index $\left(\mathrm{CD} 4^{+} \mathrm{CFSE}^{\text {lo }}\right.$ cells at background/peptide condition) of 3 , above which a responder is considered.

DQ8 restricted, suggesting that certain $\alpha$-gliadin peptides may be presented by either DQ8 or DQ2, particularly after selective deamidation.

\section{Discussion}

In the present study, we investigated peripheral $\mathrm{T}$ cell responses from young children with newly diagnosed CD prior to treatment with a gluten-free diet for two purposes: (1) develop a consistent and rapid assay to monitor peripheral $\mathrm{T}$ cell responses and (2) assess $\mathrm{T}$ cell responses to a panel of $\alpha$ - and $\gamma$-gliadin epitopes. To develop a consistent and rapid assay using limited PBMCs, $\alpha$-gliadin/DQ2 and DQ8 recombinant protein constructs were produced which have the ability to robustly stimulate six different $\mathrm{T}$ cell clones derived from adult $\mathrm{CD}$ patients, suggesting the utility of this approach with polyclonal $\mathrm{T}$ cells from the peripheral blood. However, stimulation of PBMCs from young untreated children with $\mathrm{CD}$ did not uniformly elicit $\mathrm{T}$ cell responses. There are several possible reasons for the inability to detect reactivity with these protein constructs including the possibility that $\mathrm{CD}$ donors lack $\mathrm{T}$ cells that recognize a single DQ2 or DQ8 $\alpha$-gliadin epitope. Interestingly, the double deamidated $\alpha$-gliadin epitope covalently linked to recombinant DQ8 protein failed to elicit proliferative $\mathrm{T}$ cell responses in our study population, while three individuals with proliferative responses to $\alpha$-I gliadin did not produce IFN- $\gamma$ above background levels when stimulated with the recombinant $\alpha$-I gliadin/DQ2 protein. As these epitopes were identified from adult CD patients, our data supports the heterogeneity of $\mathrm{T}$ cell responses in $\mathrm{CD}$ and that children 
TABLE 3: Overview of the T cell responses to tested DQ2 and DQ8 gliadin epitopes.

\begin{tabular}{|c|c|c|c|c|c|c|c|c|c|c|c|c|}
\hline \multirow{2}{*}{\multicolumn{2}{|c|}{ Epitopes }} & \multicolumn{6}{|c|}{$\alpha$-gliadin } & & \multicolumn{4}{|c|}{$\gamma$-gliadin } \\
\hline & & \multicolumn{4}{|c|}{ DQ8 epitopes } & \multicolumn{3}{|c|}{ DQ2 epitopes } & \multicolumn{4}{|c|}{ DQ8 epitopes } \\
\hline Case & $\begin{array}{l}\text { HLA-DQ } \\
\text { genotype }\end{array}$ & Native & $\mathrm{p} 1 \mathrm{E}$ & p9E & p1E, p9E & $\alpha-\mathrm{I}$ & $\alpha$-II & $\gamma$-I & Native & $\mathrm{p} 1 \mathrm{E}$ & p9E & p1E, p9E \\
\hline 2 & $2 / 8$ & & & & & & & & & & & \\
\hline 3 & $2 / 7$ & & & & & & & & & & & \\
\hline 4 & $2 / 2$ & & & & & & & & & & & \\
\hline 6 & $2 / 7$ & & & & & & & & & & & \\
\hline 7 & $2 / 6$ & & & & & & & & & & & \\
\hline 8 & $2 / 2$ & & & & & & & & & & & \\
\hline 9 & $2 / 6$ & & & & & & & & & & & \\
\hline 10 & $2 / 7$ & & & & & & & & & & & \\
\hline 11 & $2 / 8$ & & & & & & & & & & & \\
\hline 12 & $2 / 2$ & & & & & & & & & & & \\
\hline
\end{tabular}

Peripheral T cell responses as measured by $\mathrm{CD} 4^{+} \mathrm{CFSE}^{\text {lo }}$ proliferated cells for each individual with correlation to HLA-DQ genotype. Black boxes represent a response to the peptide with the $\mathrm{SI} \geq 3$. Gliadin epitopes are denoted as previously reported in the literature to be presented by HLA-DQ2 ( $\alpha$-I gliadin ${ }_{57-68}$ QLQPFPQPELPY, $\alpha$-II gliadin $62-73$ PQPELPYPQPQL, and $\gamma$-1 gliadin $139-152$ PEQPQQSFPEQERP) or HLA-DQ8 ( $\alpha$-gliadin $228-240$ SGQGSFQPSQQNP and $\gamma$-gliadin ${ }_{65-79}$ FPQQPQQPYPQQPQQ with and without deamidation at $\mathrm{pl}$ and $\mathrm{p} 9$ ). Three of the new-onset CD children responding to two or more peptides have the DQ2/2 genotype.

may respond to different epitopes than adults [21]. Second, the donor's $T$ cells may already be maximally stimulated as our patient population had not yet started a gluten-free diet. This is in agreement with studies reporting the need for a short-term gluten challenge in adult CD patients already on a gluten-free diet to elicit robust peripheral $\mathrm{T}$ cell responses $[8,9]$. However, peripheral $\mathrm{T}$ cell responses can exist at disease onset and disappear with a gluten-free diet. Finally, it is possible that measuring secreted IFN- $\gamma$ may not be the best way to detect responses, and other methods for detecting antigen specific $\mathrm{T}$ cell responses to gliadin may be necessary, such as using gliadin/DQ2 fluorescent tetramers [22].

In addition to evaluating peptide/MHC recombinant protein to elicit $\mathrm{T}$ cell responses, we utilized proliferation assays with bulk unfractionated PBMCs to assess peptide reactivity. Stimulation of PBMCs from newly diagnosed and untreated $\mathrm{CD}$ children is better detected using proliferation assays rather than measurement of secreted cytokine. In our panel of DQ2 and DQ8 restricted antigens, we found that $6 / 10$ children responded to at least one of the peptides tested, and the remainder did not respond to any tested peptide. It is notable that a peptide traditionally considered to be a DQ8 epitope, $\alpha$-gliadin p1E $^{228}$ SGEGSFQPSQQNP ${ }^{240}$, showed the greatest ability to stimulate $\mathrm{T}$ cells in $60 \%$ of children, even though only two children expressed the HLA-DQ8 allele (all of the children had at least one DQ2 allele). Furthermore, there were three subjects that had responses to two or more peptides and all three were DQ2/DQ2 homozygotes (Table 3). The peptide binding grooves of HLA-DQ2 and DQ8 share structural similarity with both molecules capable of anchoring peptides with acidic side chains (glutamic and aspartic acid) at pockets 1 and 9 [23]. With tissue transglutaminase present to deamidate gliadin peptides, it is plausible to hypothesize that certain deamidated epitopes have the potential to be presented by both the DQ2 and DQ8 molecules $[24,25]$. There is precedence for this concept as both HLA-DR1 and DR4 class II molecules are capable of presenting the hemagglutinin peptide, $\mathrm{HA}_{306-318}$, to $\mathrm{T}$ cells $[26,27]$.

In summary, T cell reactivity in young children with newly diagnosed and untreated $\mathrm{CD}$ is heterogeneous but favors reactivity to $\alpha$-gliadin epitopes more than $\gamma$-gliadin. It is unlikely that a single gliadin epitope will elicit $\mathrm{T}$ cell responses in all individuals and a short-term gluten challenge may be necessary to detect ample $\mathrm{T}$ cell reactivity in the peripheral circulation. However, it is possible to detect $\mathrm{T}$ cell responses without a gluten challenge or in vitro stimulus other than antigen, when measuring proliferative responses. A more comprehensive screening of gliadin epitopes in young children with newly diagnosed $\mathrm{CD}$ is necessary to identify peripheral blood $\mathrm{T}$ cell reactivity, followed by repeat assessments over time to correlate responses to disease activity and treatment with a gluten-free diet. Monitoring peripheral $\mathrm{T}$ cell responses to gliadin epitopes present at disease onset, which can disappear with a gluten-free diet, has clinical utility in identifying cases of refractory $\mathrm{CD}$ or in assessing the effectiveness of emerging therapies for $\mathrm{CD}$ treatment.

\section{Conflict of Interests}

The authors E. Liu, K. McDaniel, S. Case, L. Yu, and A. W. Michels have no conflict of interests regarding the publication of this paper. The authors B. Gerhartz, N. Ostermann, G. Fankhauser, V. Hungerford, C. Zou, M. Luyten, and K. J. Seidl are all employees at Novartis and may own Novartis stock.

\section{Acknowledgments}

This work was supported by Grants from the National Institute of Diabetes and Digestive Kidney Diseases (R01 DK032083 and K08 DK095995) and the Celiac Disease Center at the Children's Hospital Colorado. The authors would 
like to thank Ludvig Sollid for providing DNA constructs of the $\mathrm{T}$ cell receptor from gliadin responsive $\mathrm{T}$ cell clones.

\section{References}

[1] P. H. R. Green and C. Cellier, "Medical progress: celiac disease," The New England Journal of Medicine, vol. 357, no. 17, pp. 17311743, 2007.

[2] L.-E. Fallang, E. Bergseng, K. Hotta, A. Berg-Larsen, C.-Y. Kim, and L. M. Sollid, "Differences in the risk of celiac disease associated with HLA-DQ2.5 or HLA-DQ2.2 are related to sustained gluten antigen presentation," Nature Immunology, vol. 10, no. 10, pp. 1096-1101, 2009.

[3] L. Shan, Ø. Molberg, I. Parrot et al., "Structural basis for gluten intolerance in Celiac Sprue," Science, vol. 297, no. 5590, pp. 2275-2279, 2002.

[4] M. F. Kagnoff, "Celiac disease: pathogenesis of a model immunogenetic disease," Journal of Clinical Investigation, vol. 117, no. 1, pp. 41-49, 2007.

[5] V. Abadie, L. M. Sollid, L. B. Barreiro, and B. Jabri, "Integration of genetic and immunological insights into a model of celiac disease pathogenesis," Annual Review of Immunology, vol. 29, pp. 493-525, 2011.

[6] S. Guandalini and M. Setty, "Celiac disease," Current Opinion in Gastroenterology, vol. 24, no. 6, pp. 707-712, 2008.

[7] Ø. Molberg, S. N. Mcadam, R. Körner et al., "Tissue transglutaminase selectively modifies gliadin peptides that are recognized by gut-derived T cells in celiac disease," Nature Medicine, vol. 4, no. 6, pp. 713-717, 1998.

[8] R. P. Anderson, P. Degano, A. J. Godkin, D. P. Jewell, and A. V. S. Hill, "In vivo antigen challenge in celiac disease identifies a single transglutaminase-modified peptide as the dominant Agliadin T-cell epitope," Nature Medicine, vol. 6, no. 3, pp. 337342, 2000.

[9] R. P. Anderson, D. A. van Heel, J. A. Tye-Din et al., "T cells in peripheral blood after gluten challenge in coeliac disease," Gut, vol. 54, no. 9, pp. 1217-1223, 2005.

[10] J. O’Keeffe, K. Mills, J. Jackson, and C. Feighery, “T cell proliferation, MHC class II restriction and cytokine products of gliadin-stimulated peripheral blood mononuclear cells (PBMC)," Clinical and Experimental Immunology, vol. 117, no. 2, pp. 269-276, 1999.

[11] J. A. Tye-Din, J. A. Stewart, J. A. Dromey et al., "Comprehensive, quantitative mapping of $\mathrm{T}$ cell epitopes in gluten in celiac disease," Science Translational Medicine, vol. 2, no. 41, Article ID 41ra51, 2010.

[12] S. Tollefsen, H. Arentz-Hansen, B. Fleckenstein et al., "HLADQ2 and -DQ8 signatures of gluten T cell epitopes in celiac disease," Journal of Clinical Investigation, vol. 116, no. 8, pp. 2226-2236, 2006.

[13] S. E. Broughton, J. Petersen, A. Theodossis et al., "Biased T cell receptor usage directed against human leukocyte antigen DQ8restricted gliadin peptides is associated with celiac disease," Immunity, vol. 37, no. 4, pp. 611-621, 2012.

[14] M. Li, L. Yu, C. Tiberti et al., "A report on the international transglutaminase autoantibody workshop for celiac disease," American Journal of Gastroenterology, vol. 104, no. 1, pp. 154163, 2009.

[15] T. L. Bugawan and H. A. Erlich, "Rapid typing of HLA-DQB1 DNA polymorphism using nonradioactive oligonucleotide probes and amplified DNA," Immunogenetics, vol. 33, no. 3, pp. 163-170, 1991.
[16] A. W. Michels, D. A. Ostrov, L. Zhang et al., "Structure-based selection of small molecules to alter allele-specific MHC class II antigen presentation," Journal of Immunology, vol. 187, no. 11, pp. 5921-5930, 2011.

[17] J. Holst, A. L. Szymczak-Workman, K. M. Vignali, A. R. Burton, C. J. Workman, and D. A. Vignali, "Generation of T-cell receptor retrogenic mice," Nature Protocols, vol. 1, no. 1, pp. 406-417, 2006.

[18] J. P. Scott-Browne, J. White, J. W. Kappler, L. Gapin, and P. Marrack, "Germline-encoded amino acids in the $\alpha \mathrm{B}$ T-cell receptor control thymic selection," Nature, vol. 458, no. 7241, pp. 1043-1046, 2009.

[19] H. Sjöström, K. E. A. Lundin, Ø. Molberg et al., "Identification of a gliadin T-cell epitope in coeliac disease: general importance of gliadin deamidation for intestinal T-cell recognition," Scandinavian Journal of Immunology, vol. 48, no. 2, pp. 111-115, 1998.

[20] H. Arentz-Hansen, R. Körner, Ø. Molberg et al., “The intestinal $\mathrm{T}$ cell response to $\alpha$-gliadin in adult celiac disease is focused on a single deamidated glutamine targeted by tissue transglutaminase," The Journal of Experimental Medicine, vol. 191, no. 4, pp. 603-612, 2000.

[21] W. Vader, Y. Kooy, P. van Veelen et al., "The Gluten response in children with celiac disease is directed toward multiple gliadin and glutenin peptides," Gastroenterology, vol. 122, no. 7, pp. 1729-1737, 2002.

[22] H. Quarsten, S. N. McAdam, T. Jensen et al., "Staining of celiac disease-relevant T cells by peptide-DQ2 multimers," Journal of Immunology, vol. 167, no. 9, pp. 4861-4868, 2001.

[23] E. Y. Jones, L. Fugger, J. L. Strominger, and C. Siebold, "MHC class II proteins and disease: a structural perspective," Nature Reviews Immunology, vol. 6, no. 4, pp. 271-282, 2006.

[24] K. N. Henderson, J. A. Tye-Din, H. H. Reid et al., "A structural and immunological basis for the role of human leukocyte antigen DQ8 in celiac disease," Immunity, vol. 27, no. 1, pp. 2334, 2007.

[25] K. N. Henderson, H. H. Reid, N. A. Borg et al., "The production and crystallization of the human leukocyte antigen class II molecules HLA-DQ2 and HLA-DQ8 complexed with deamidated gliadin peptides implicated in coeliac disease," Acta Crystallographica Section F, vol. 63, part 12, pp. 1021-1025, 2007.

[26] J. V. Brawley and P. Concannon, "Complementarity-determining region 1 sequence requirements drive limited $\mathrm{V} \alpha$ usage in response to influenza hemagglutinin 307-319 peptide," Journal of Immunology, vol. 168, no. 8, pp. 3894-3901, 2002.

[27] J. Hennecke and D. C. Wiley, "Structure of a complex of the human $\alpha / \beta$ T cell receptor (TCR) HA1.7, Influenza hemagglutinin peptide, and major histocompatibility complex class II molecule, HLA-DR4 (DRA $* 0101$ and DRBI*0401): insight into TCR cross-restriction and alloreactivity," The Journal of Experimental Medicine, vol. 195, no. 5, pp. 571-581, 2002. 


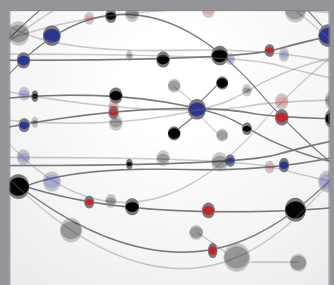

The Scientific World Journal
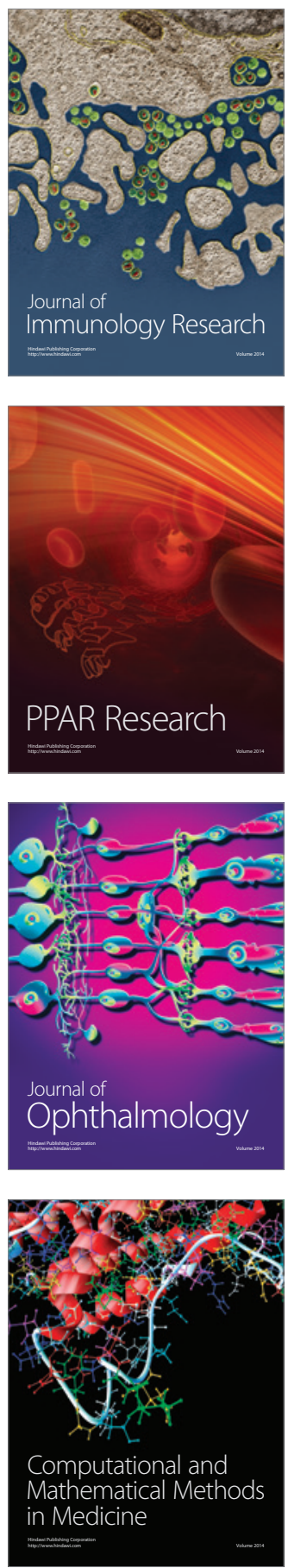

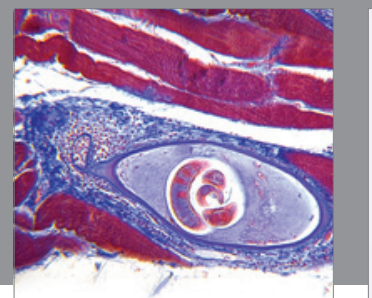

Gastroenterology

Research and Practice
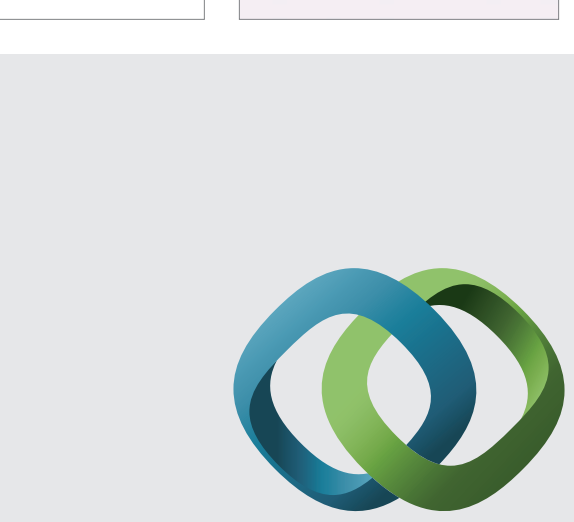

\section{Hindawi}

Submit your manuscripts at

http://www.hindawi.com
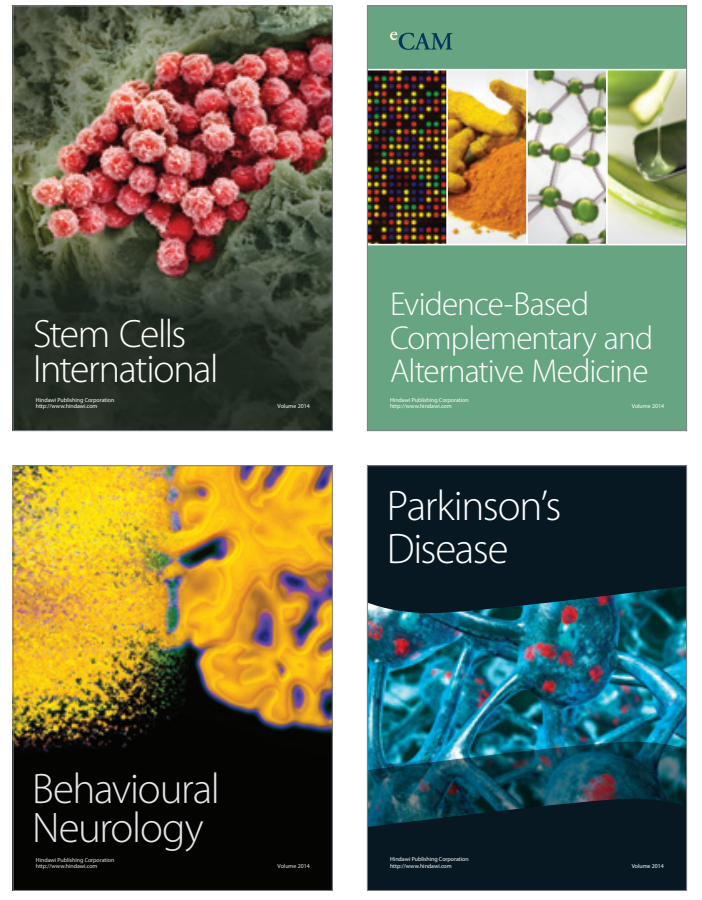
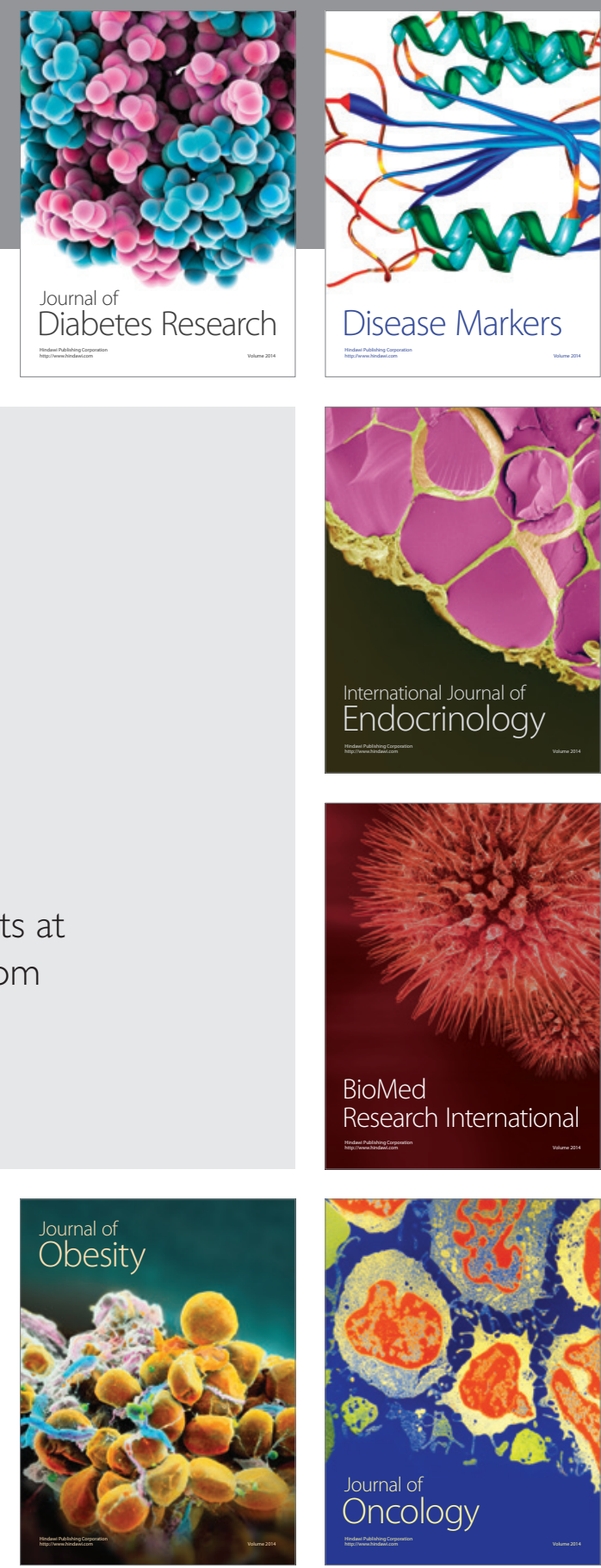

Disease Markers
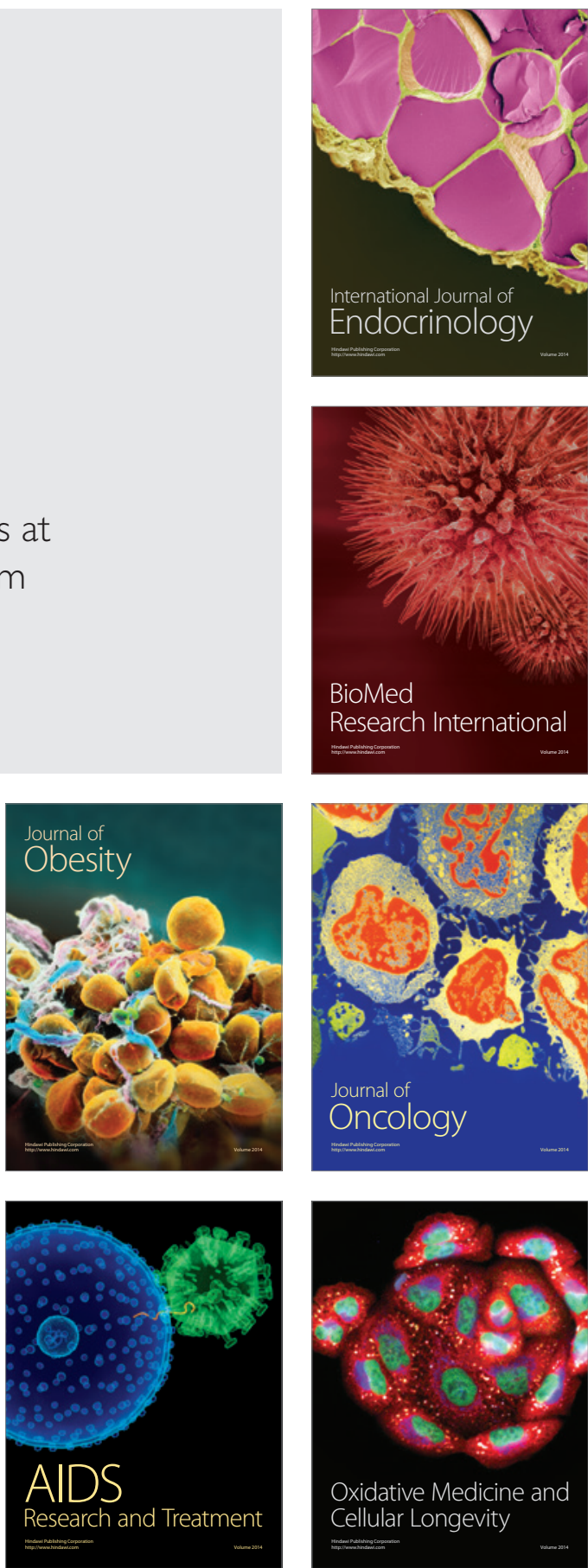